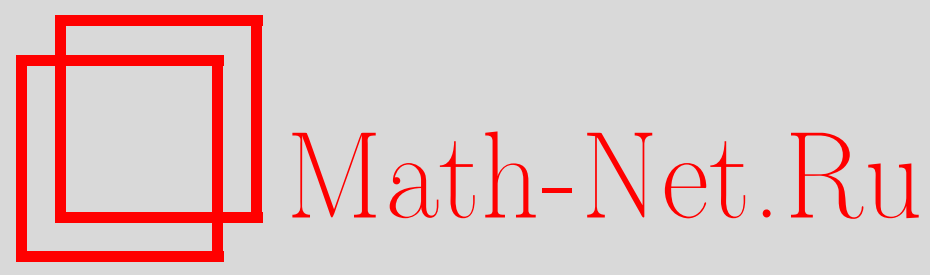

И. В. Протасов, Разбиения групп на большие подмножества, Матем. заметки, 2003, том 73, выпуск 3, 471-473

DOI: https://doi.org/10.4213/mzm621

Использование Общероссийского математического портала Math-Net.Ru подразумевает, что вы прочитали и согласны с пользовательским соглашением http://www. mathnet.ru/rus/agreement

Параметры загрузки:

IP: 3.85 .73 .92

26 апреля 2023 г., 10:55:41

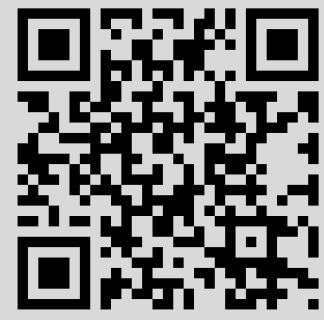




\section{РАЗБИЕНИЯ ГРУПП НА БОЛЬШИЕ ПОДМНОЖЕСТВА}

\section{И.В. Протасов}

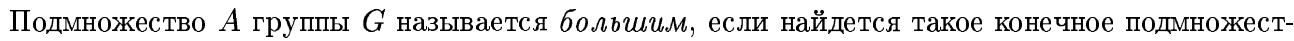
во $F$, что $G=F A=A F$. В статье [1] А. Белла и В. И. Малыхин поставили следующий вопрос.

Можно ли любую бесконечную группу разбить на два большие подмножсества?

Цель заметки - доказать следующую теорему.

Теорема. Любую бесконечную группу можно разбить на счетное число больших подмножеств.

Схема доказательства такова. Вначале строится разбиение группы на счетное число болшши подмножеств при условии, что на группе можно указать некоторое счетное семейство разбиений на конечные подмножества. Затем доказывается, что каждая бесконечная группа допускает такое семейство разбиений.

Семейство $\left\{\mathfrak{R}_{n}, n \in \omega\right\}$ разбиений группы $G$ на конечные подмножества назовем $n p a в и л ь н ы м$, если для любого $n \in \omega$ выполнены следующие условия:

1) все подмножества разбиения $\Re_{n}$ имеют равное число элементов;

2 ) разбиение $\Re_{n+1}$ является укрупнением разбиения $\Re_{n}$, т.е. каждое подмножество разбиения $\Re_{n+1}$ является объединением $\geqslant 2$ подмножеств разбиения $\mathfrak{R}_{n}$;

3 ) найдется такое конечное подмножество $F_{n}$, что $A \subseteq F_{n} x$ для всех $A \in \Re_{n}, x \in A$.

ЛЕмма 1. Если әруппа $G$ допускает правильное семейство разбиений $\left\{\Re_{n}, n \in \omega\right\}$, то $G$ можно разбить на счетное число больших подмножеств.

ДоКАЗАТЕЛЬСтво. Можносчитать, что подмножества разбиения $\mathfrak{R}_{0}$ неодноэлементны. Положим $L_{0}=\left\{A^{-1}: A \in L_{0}\right\}$ и рассмотрим пару разбиений $\mathfrak{R}_{0}, L_{0}$. По теореме об общей трансверсали $\left[2\right.$, теорема 7.4.4] найдется такое подмножество $B \subset G$, что $\left|A \cap B_{0}\right|=\left|A^{-1} \cap B_{0}\right|=1$ для всех подмножеств $A \in \mathfrak{R}_{0}$. По условию 3) $F_{0} B_{0}=G, B_{0} F_{0}^{-1}=G$. Следовательно, $\left(F_{0} \cup F_{0}^{-1}\right) B_{0}=$ $B_{0}\left(F_{0} \cup F_{0}^{-1}\right)$ и подмножество $B_{0}$ большое. Положим $\mathfrak{R}_{1}^{\prime}=\left\{A \backslash B_{0}: A \in \mathfrak{R}_{1}\right\}, L_{1}^{\prime}=\left\{R^{-1}:\right.$ $\left.R \in \mathfrak{R}_{1}\right\}$. По условиям 1), 2) все подмножества разбиений $\mathfrak{R}_{1}^{\prime}$ и $L_{1}^{\prime}$ множества $G \backslash B_{0}$ непустые и содержат одинаковое число элементов. Применим теорему об общей трансверсали к разбиениям $\mathfrak{R}_{1}^{\prime}, L_{1}^{\prime}$ и выберем подмножество $B_{1} \subset G \backslash B_{0}$ такое, что $\left|A \cap B_{1}\right|=\left|A^{-1} \cap B_{1}\right|=1$ для всех $A \in \mathfrak{R}_{1}^{\prime}$. По условию 3) $F_{1} B_{1}=B_{1} F_{1}^{-1}=G$. Положим $\mathfrak{R}_{2}^{\prime}=\left\{A \backslash\left(B_{0} \cup B_{1}\right): A \in \mathfrak{R}_{2}\right\}$, $L_{2}^{\prime}=\left\{R^{-1}: R \in \mathfrak{R}_{2}\right\}$ и построим соответствующую трансверсаль $B_{2}$. Условия 1$)$, 2) гарантируют возможность продолжить этот процесс счетное число шагов, а условие 3 ) показывает, что все подмножества построенного дизъюнктного семейства $\left\{B_{n}, n \in \omega\right\}$ большие. Присоединим к одному из этих подмножеств $G \backslash \bigcup_{n \in \omega} B_{n}$; получим искомое разбиение группы.

Лемма 2. Если в группе $G$ существует возрастающая иепь $H_{0} \subset H_{1} \subset \cdots \subset H_{n} \subset \cdots$ конечных подгрупп, то $G$ допускает правильное семейство разбиений.

ДокАЗАтЕЛЬСтво. Достаточно взять в качестве $\mathfrak{R}_{n}$ разбиение группы $G$ на правые смежные классы по подгруппе $H_{n}$ и положить $F_{n}=H_{n}$.

Для построения правильного семейства разбиений на группе, содержащей бесконечную конечно порожденную подгруппу, нам понадобится специальная нумерация множества вершин графа.

Пусть $G r=(V, E)$ - связньй граф̆ с множеством вершин $V$ и множеством ребер $E, E \subseteq V \times$ $V, E^{-1}=E, E^{-1}=\{(y, x):(x, y) \in E\}$. Для произвольных вершин $x, y \in V$ определим число $d(x, y)$ как длину кратчайшего пути между $x$ и $y$. Положим также $B(x, m)=\{y \in d(x, y) \leqslant$ $m\}, x \in V, m \in \omega$.

ЛЕмма 3. Пусть $G r=(V, E)$ - конечный связный граф, $|V|=n, x, y \in V, x \neq y$ u $(x, y) \in E$. Тогда существует биекиия $f:\{1,2, \ldots, n\} \rightarrow V$ такая, что $f(1)=x, f(n)=y$ $u d(f(i), f(i+1)) \leqslant 3$ для всех $i \in\{1,2, \ldots, n-1\}$. 
ДоКАЗАТЕльство. Заменяя граф $G r$ его остовом, можно считать, что граф $G r$ является деревом. Применим индукцию по числу $n$. Для $n=2$ утверждение очевидно. Предположим, что $n>2$ и рассмотрим два случая.

Случай $1 .|B(x, 1)|=2$, т.е. $B(x, 1)=\{x, y\}$. Удаляя вершину $x$ и ребро $(x, y)$, получим дерево $G r^{\prime}=\left(V^{\prime}, E^{\prime}\right)$, где $V^{\prime}=V \backslash\{x\}, E^{\prime}=E \backslash\{(x, y)\}$. Так как $|V| \geqslant 2$, существует такая вершина $z \in V^{\prime}$, что $(y, z) \in V^{\prime}$. По предположению индукции существует биекция $f^{\prime}:\{2,3, \ldots, n\} \rightarrow V^{\prime}$ такая, что $f^{\prime}(z)=2, f^{\prime}(y)=n$ и $d\left(f^{\prime}(i), f^{\prime}(i+1)\right) \leqslant 3$ для всех $i \in\{2,3, \ldots, n-1\}$. Определим биекцию $f:\{1,2 \ldots, n\} \rightarrow V$ по правилу $f(1)=x$ и $f(i)=f^{\prime}(i)$ для всех $i \in\{2,3, \ldots, n\}$. Так как $d(x, z)=2$, то $f$ - искомая нумерация.

Случай 2. $|B(x, 1)|>2$. Если $|B(y, 1)|=2$, то заменой пары $x, y$ на $y, x$ возвращаемся к первому случаю. Таким образом, $|B(x, 1)|>2$ и $|B(y, 1)|>2$. Удаляя ребро $(x, y)$, получим два дерева $G r_{1}=\left(V_{1}, E_{1}\right)$ и $G r_{2}=\left(V_{2}, E_{2}\right), x \in V_{1}, y \in V_{2}$. Пусть $\left|V_{1}\right|=k, k<n$.

Выберем произвольную вершину $x^{\prime} \in V_{1}$, смежную с вершиной $x$. По предположению индукции существует биекция $f_{1}:\{1,2, \ldots, k\} \rightarrow V_{1}$ такая, что $f_{1}(1)=x, f_{1}(k)=x^{\prime}$ и $d\left(f_{1}(i), f_{1}(i+1)\right) \leqslant 3$ для всех $i \in\{2,3, \ldots, k-1\}$.

Выберем произвольную вершину $y^{\prime} \in V_{2}$, смежную с вершиной $y$. По предположению индукции существует биекция $f_{2}:\{k+1, k+2, \ldots, n\} \rightarrow V_{2}$ такая, что $f_{2}(k+1)=y^{\prime}, f_{2}(n)=y$ и $d\left(f_{2}(i), f_{2}(i+1)\right) \leqslant 3$ для всех $i \in\{k+1, k+2, \ldots, n-1\}$.

Наконец, определим биекцию $f:\{1,2, \ldots, n\} \rightarrow V$ по правилу $f(i)=f_{1}(i), i \in\{1,2, \ldots, k\}$ и $f(i)=f_{2}(i), i \in\{k+1, k+2, \ldots, n\}$. Так как расстояние между $x^{\prime}$ и $y^{\prime}$ в графе $G r$ равно 3 , то $f-$ искомая биекция.

Бесконечное дерево $T r=(V, E)$ назовем одноствольным, если найдется такое счетное подмножество вершин $V^{\prime}=\left\{v_{n}: n \in \omega\right\}$, что $\left(v_{n}, v_{n+1}\right) \in E, n \in \omega$ и после стирания ребер $E^{\prime}=\left\{\left(v_{n}, v_{n+1}\right): n \in \omega\right\}$ дерево $\operatorname{Tr}$ распадается на конечные деревья. Подграф̆ $\left(V^{\prime}, E^{\prime}\right)$ называется стволом дерева $\mathrm{Tr}$.

Лемма 4. Пусть $\mathrm{Tr}=(V, E)$ - одноствольное дерево. Тогда существует биекиия $f:\{1,2, \ldots\} \rightarrow V$ maкая, что $d(f(i), f(i+1)) \leqslant 3$ для всех $i \in\{1,2, \ldots\}$.

ДоказАтельство. Пусть $\left(V^{\prime}, E^{\prime}\right)$ - ствол дерева $T r, V^{\prime}=\left\{v_{n}: n \in \omega\right\}, E^{\prime}=\left\{\left(v_{n}, v_{n+1}\right)\right.$ : $n \in \omega\}$. Тогда граф $\left(V, E \backslash E^{\prime}\right)$ является дизъюнктньм объединением конечных деревьев $\operatorname{Tr}_{n}=$ $\left(V_{n}, E_{n}\right), v_{n} \in V_{n}$. Если $\left|V_{n}\right|=1$, положим $v_{n}^{\prime}=v_{n}$. Иначе, выберем любую вершину $v_{n}^{\prime} \in V_{n}$, смежную в графе $T r_{n}$ с вершиной $v_{n}$. Обозначим $k_{n}=\left|V_{n}\right|, n \in \omega$. По лемме 3 существуют биекции

$$
\begin{aligned}
& f_{0}:\left\{1,2, \ldots, k_{0}\right\} \rightarrow V_{0}, \quad f_{0}\left(v_{0}^{\prime}\right)=1, \quad f_{0}\left(v_{0}\right)=k_{0}, \\
& f_{1}:\left\{k_{0}+1, k_{0}+2, \ldots, k_{0}+k_{1}\right\} \rightarrow V_{1}, \quad f_{1}\left(v_{1}^{\prime}\right)=k_{0}+1, \quad f_{1}\left(v_{1}\right)=k_{0}+k_{1}, \\
& f_{2}:\left\{k_{0}+k_{1}+1, k_{0}+k_{1}+2, \ldots, k_{0}+k_{1}+k_{2}\right\} \rightarrow V_{2}, \\
& \quad f_{2}\left(v_{2}^{\prime}\right)=k_{0}+k_{1}+1, \quad f_{2}\left(v_{2}\right)=k_{0}+k_{1}+k_{2},
\end{aligned}
$$

удовлетворяюшие условию $d\left(f_{n}(i), f_{n}(i+1)\right) \leqslant 3$ для всех $n \in \omega, i \in\left\{k_{0}+k_{1}+\cdots+k_{n-1}+1, \ldots\right.$, $\left.k_{0}+k_{1}+\cdots+k_{n}\right\}$.

Определим биекцию $f:\{1,2, \ldots\} \rightarrow V$, полагая $f(i)=f_{n}(i)$ тогда и только тогда, когда $i$ попадает в область определения функции $f_{n}$. Поскольку расстояние в графе $\operatorname{Tr}$ между вершинами $v_{n}$ и $v_{n+1}^{\prime}$ не превосходит $2, f$ - искомая нумерация.

ЛЕмма 5. Если әруппа $G$ содержит бесконечную конечно порохденную подгруппу $H$, то $G$ допускает правильное семейство разбиений.

ДокАЗАтЕльство. Пусть $S$ - конечная система образующих группы $H, S=S^{-1}$. Рассмотрим граф̆ Кэли Сау группы $H$. Вершинами графа Сау являются элементы группы $H$, а вершины $x$, $y$ соединены ребром $(x, y)$ тогда и толко тогда, когда $x^{-1} y \in S$. Заметим, что граф Сау связен и выделим его остов $(G, E)=T r$. Пользуясь леммой Кенига о существовании бесконечной цепи в любом бесконечном локальн конечном дереве, множество $G$ вершин дерева $\operatorname{Tr}$ можно разбить на 
счетные подмножества так, что для любого подмножества $A$ разбиения граф $G r(A)=(A, E(A))$, $E(A)=E \cap(A \times A)$, является одноствольным деревом. По лемме 4 существует биекция $f_{A}:\{1$, $2, \ldots\} \rightarrow A$ такая, что $d\left(f_{A}(i), f_{A}(i+1)\right) \leqslant 3$ для всех $i \in \omega$. Определим счетное семейство разбиений $\left\{\mathfrak{R}_{n}^{\prime}(A), n \in \omega\right\}$ подмножества $A$, полагая

$$
\mathfrak{R}_{n}^{\prime}(A)=\left\{f_{A}\left(\left\{1,2, \ldots, 2^{n}\right\}\right), f_{A}\left(\left\{2^{n}+1,2^{n}+2, \ldots, 2^{n+1}\right\}\right), \ldots\right\} .
$$

Положим $m=3 \cdot 2^{n}$ и заметим, что

$$
f_{A}(R) \subseteq S^{m} f_{A}(i)
$$

для любого подмножества $R$ из разбиения $\mathfrak{R}_{n}^{\prime}(A)$ и любого элемента $f_{A}(i) \in R$.

Объединяя разбиения $\mathfrak{R}_{n}^{\prime}(A)$ по всем подмножествам $A$ разбиения графа $T r$ на одноствольные деревья, получим некоторое разбиение $\mathfrak{R}_{n}^{\prime}$ подгруппы $H$. Выберем некоторое множество $X$ представителей правых смежных классов группы $G$ по подгруппе $H$. Положим $\mathfrak{R}_{n}=\left\{R x: R \in \mathfrak{R}_{n}^{\prime}\right.$, $x \in X\}$. Очевидно, что семейство разбиений $\left\{\Re_{n}, n \in \omega\right\}$ удовлетворяет условиям 1$), 2$ ) определения правильного семейства разбиений, а условие 3 ) следует из соотношения (*).

ДОКАЗАТЕЛЬСТВО ТЕОРЕМЫ следует непосредственно из лемм $1,2,5$.

Можно ли произвольню бесконечную группу мощности $k$ разбить на $K$ больших подмножеств? Ответ отрицательный и очевидное препятствие - аменабельность. В аменабельной группе каждое большое подмножество имеет положительню банахову меру, а поэтому любое дизъюнктное семейство больших подмножеств не более чем счетно. В статье [1] поставлен следующий вопрос.

Существует ли группа, которую мохно разбить на несчетное число больших подмножеств?

Ответ на этот вопрос утвердительньй. Покажем, что свободную группу ранга $k$ можно разбить на $k$ большшх подмножеств.

Пусть $F(X)$ - свободная группа в алфавите $X,|X|=k$. Вначале укажем разбиение $F(X)$ на $k$ подмножеств больших слева. При этом подмножество $A$ группы $G$ называется большим слев $a$ $($ справ $a)$, если найдется такое конечное подмножество $F$, что $G=F A(G=A F)$. Для каждого элемента $x \in X$ обозначим через $S(x)$ множество всех групповых слов, которые начинаются с $x$ либо $x^{-1}$. Очевидно, что $F(X)=x S(x) \cup S(x)$ и $\{S(x): x \in X\}$ - дизъюнктное семейство больших слева подмножеств группы $F(X)$. Далее, возьмем произвольную собственную инвариантную подгруппу $H$ группы $G$ конечного индекса. Так как $H$ свободна, по доказанному имеется дизъюнктная система $L$ мощности $k$ больших слева в $H$ подмножеств. Выберем произвольньй элемент $g \in G \backslash H$. Тогда $\left\{A \cup g A^{-1}: A \in L\right\}$ - дизъюнктная система больших подмножеств группы $F(X)$.

\section{СПИСОК ЦИТИРОВАННОЙ ЛИТЕРАТУРЫ}

1. Bella A., Malykhin V. I. // Questions Answers Gen. Topology. 1999. V. 11. P. 183-187. 2. Орэ О. Теория графов. М.: Наука, 1980. 